\title{
Advance Computer Efficient Streaming and Sharing in the Clouds
}

\author{
Nitesh Chainani ${ }^{1}$, Kishor Phapale ${ }^{2}$, Roshan Pawar $^{3}$, Sagar Deshmukh ${ }^{4}$, Prof. N.A. Dhawas ${ }^{5}$ \\ B.E Final year, Sinhgad Institute of Technology, Lonavala, Maharashtra, India ${ }^{1,2,3,4}$ \\ professor, Sinhgad Institute of Technology, Lonavala, Maharashtra, India ${ }^{5}$
}

\begin{abstract}
Now days on video traffic over the computer networks have been sourcing, the wireless link capacity cannot keep up with the requirements of traffics. The difference between the traffic requirements and thelink capacity, along with time-changing link conditions, results in less service quality of video streaming over computer networks such as long buffering time and interruption. Usingthe cloud computing technology, we propose a new computer video streaming framework, dubbed ACES-Cloud, which divides in two types one for adaptive streaming and second is for sharing of videos.In traditional systems the performance of video was poor, toincreases the quality of video streaming the way of using ACES cloud. In this system of ACES cloud uses a technics as SVC for creating a private agent to fetching a videos from VSP and stored in the ACES cloud. Using this way the problem of traffic and so buffering problem to be solved.This paper includes the performing of various methods and structures which are used in cloud to provide effective solution for providing better service to the users.
\end{abstract}

Keywords: ACES cloud, SVC (Scalable Video Coding), SIN, ACS Algorithm.

\section{INTRODUCTION}

Cloud computing assuring low costs, higher quality, easier Maintenance, anytime. A main factor in moving to the cloud is to ensure and build confidence that user data is handled securely and easily in the cloud.A recent Microsoft survey found that "...55\% of the public and $87 \%$ of business leaders are excited about the possibilities of cloud computing. But, more than $90 \%$ of them are worried nearbyretreat, handiness, besidesisolation of successor data as it rests in the cloud."

There are severalproblems between user data protection and richcomputation in the cloud. User wants to maintain control of their data, but also want to advantage from rich services provided by application developers using that data. At present, there is tiny platform-level support and standardization for provable data protection in the cloud. Over the earlieryears, more and more traffic is accounted by video running and transferring. In particular, video streaming services over mobile networks have become prevalent over the past few years. While the video streaming is not so challenging in wired networks, computer networks have been suffering from video traffic transmissions over scarce bandwidth of wireless links. Worried efforts to enhance the wireless link bandwidth (e.g., 3G and LTE), soaring video traffic demands from mobile users are rapidly overpowering the wireless link capacity. The main matters faced during the study of video streaming and sharing achieved in mobile users under cloud environment are high traffic rate, extended buffering time, and trouble due to limited bandwidth. The study shows the usage of video or any kind of multimedia has improved over the period of years, many issues had occurred and resolved through various techniques during the traditional change happened between developing technologies.
Lately there have been many studies on how to progress the service quality of Computer video streaming on two aspects:[1]

1. Scalability: Computer video streaming services should support a wide spectrum of mobile devices; they have different video resolutions, diverse computing powers, diverse wireless links (like $3 \mathrm{G}$ and LTE) and so on. Also, the available link size of a mobile device may vary over time and space depending on its signal strength, other user's traffic in the same cell, and link condition variation.

2. Adaptability: Traditional video streaming techniques designed by considering relatively stable traffic links between servers and users perform poorly in mobile environments [1]. Thus the fluctuating wireless link status should be properly dealt with to provide _tolerablell video streaming services. To address this issue, we have to adjust the video bit rate adapting to the currently timevarying available link bandwidth of each mobile user. Such adaptive streaming techniques can effectively reduce packet losses and bandwidth waste.

This paper shows the 3 type of design modules: one for the admin, second for the user1, third for the user2.Admin module provides functionality upload videos, download videos, Storing the videos and authentication. User module provided the uploading, downloading, sharing and request to another user.CDN is a traditional solution based on deploying servers at the edge of the network, near video access points. Scalability is a limitation of CDN because the server capacity becomes a bottleneck when there are a large number of concurrent peer requests. So to overcome these problems we introduced ACES Clouds. 


\section{RELATED wORK}

I. Streaming and Sharing of Videos in Computer Network A.Cloud Computing Technique:

Cloud computing techniques are used to flexibly providescalable possessions to content, package providers, and process offloading to computer users. Thus, cloud data centers can easily provision for large-scale real-time video services as. Several studies on Computer cloud computing technologies have proposed to generate personalized intelligent agents for servicing computer users, hence, in the cloud, multiple agent instances or multithreads can be maintained dynamically and efficiently depending on the time-varying user demands. SVC is an extension to the H.264/AVC standard. It is secret as a layered video codec which can encode a video stream in several types and numbers of development layers on top of the AVC /H.264compatible base layer. These development layers can be added or removed from the bit stream during streaming without re-encoding of the media. The transmission speed of accessible video streams in the computer network can be controlled by using TCP friendly rate control. The streams are encoded using the SVC delayof the H.264/AVC standard. TFRC during variable channel processing power, system resources, and network A number of protocols for service level concession have been proposed, such as common open policy service for service level specification (COPS-SLS) [4], resource negotiation and pricing protocol (RNAP) [5], and service negotiation protocol (SrNP) [6]. Additionally, two protocols have been offered to support QoS negotiation in wireless networks by considering users mobility, namely, QoS basic signaling layer protocol (QoS) [7] and dynamic service concession protocol (DSNP)[8]. QoS GSLP uses mobility and traffic pattern guess to predict the next point of attachment of a mobile user and delivers negotiation interval. This method highly increases the complexityIn this mechanism, after a user negotiates its service.

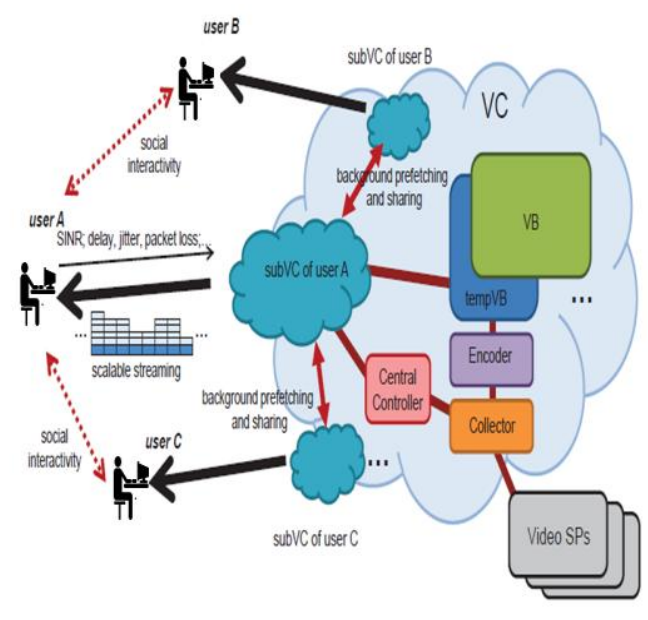

Fig 1 ACES Architecture

\section{AMES-CLOUD FRAMEWORK}

In this section we explain the ACES-Cloud framework includes the Adaptive Computer Video streaming $(\mathrm{ACoV})$ and the Efficient Social Video sharing (ESoV).
As shown in Fig. 1[2], the whole video storing and streaming system in the cloud is called the Video Cloud (VC).In the VC, there is a large-scale video base (VB), which stores the most of the common video clips for the videoservice providers (VSPs). A temporal video base (tempVB) is used to cache new candidates for the common videos, while tempVB counts the access frequency of each video. The VC keeps running a collector to pursue videos whichare already standard in VSPs, and will re-encode the collected videos into SVC format and store into tempVB first. By this 2-tier storage, the ACES-Cloud can keep serving most of popular videos eternally. Note that managementwork will be handled by the controller in the VC.Specific for each computer user, a sub-video cloud (subVC) is created dynamically if there is any video streaming.

Note that the video deliveries among the subVCs and the VC in most cases are actually not "copy", but just "link" operations on the same file eternally within the cloud data center [3]. There is also encoding function in subVC(actually a smaller-scale encoder instance of the encoder in VC), and if the mobile user burdens a new video,which is not in the subVB or the VB in VC, the subVC will realize, encrypt and relocation the video. During videoflooding, mobile users will always report link conditions to their corresponding subVCs, and then the subVCsoffer adaptive video streams. Note that each mobile device also has a temporary caching storage, which is calledlocal video base (localVB), and is used for buffering and prefetching.Note that as the cloud service may crosswaysdiverse places, or even regions, so in the case of a video deliveryand prefetching between different data midpoints, an transmission will be carried out, which can be then called "copy". And because of the best deployment of documents centers, as well as the capable links among the data midpoints, the "copy" a large video file takes minuteinterval.

\section{ACOV: ADAPTIVE Computer VIDEO STREAMING} A. SVC

As shown in Fig. 2, traditional video streams with fixed bit rates cannot adapt to the fluctuation of the linkvalue. For a individual bit rate, if the supportable link bandwidth differs much, the video flooding can be frequentlycompleted due to the packet loss.[10]

IV. Matching Algorithm between BW and Segments

$\mathrm{i}=0$

$\mathrm{BW} 0=\mathrm{RBL}$

Transmit BL0

Monitor BW0

practical

repeat

Sleep for Twin

Obtain pi, RTTi, SINRi etc., from client's report

Predict BW i+1

estimate (or BW i+1

estimate $=\mathrm{BWi}$

practical) 

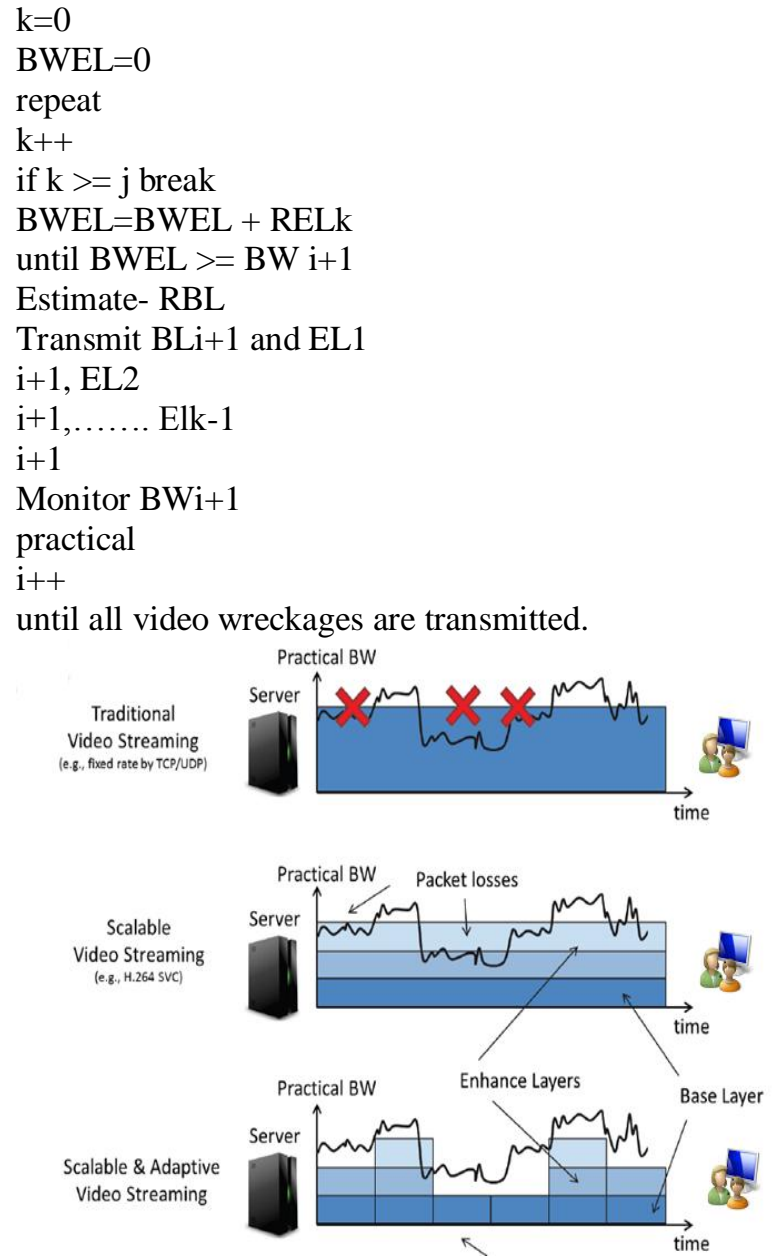

Fig. 2. A comparison of the traditional video streaming, scalable video streaming and theadaptive video streaming in the ACES-Cloud structure.

In SVC, a combination of the three lowest scalability is called the Base Layer (BL) while the enhancedcombinations are called Enhancement Layers (ELs). To this regard, if BL is guaranteed to be distributed, whilemore ELs can be also gained when the link can give, a better video value can be ordinary.

\section{EXISTINGSYSTEM}

In Existing System adaptive mobile video flooding and allocation framework, called ACES-Cloud, which efficiently stores videos in the clouds (VC), and utilizes cloud computing to construct private agent (subVC) for each mobile user to try to offer "non-terminating" video streaming adapting to the fluctuation of link quality based on the Scalable Video Coding method. Also ACES-Cloud can further seek to deliver "nonbuffering"experience of video streaming by background pushing functions among the VB, subVBs and localVB of computer users. We estimated the ACES-Cloud by prototype implementation and shows that the cloud computing technique brings significant improvement on the adaptivity of the mobile streaming. We snubbed the cost of encoding workload in the cloud while implementing the prototype.

\section{IV.PROPOSED SYSTEM}

In this paper we proposed that streaming and sharing of videos in the computer with the help of clouds. In Existing system there are difference problem we will faced like Memory issue, Integration, Technical issue. We can overcomethat entire problem with the help of matching algorithm and subVc cloud. In that we improve the Scalability, Reliability and also we can share the video easily and also send this video to the other clients. One client can access all the video to other clients.

\section{IMPLEMENTATION}

Implementation is the stage of the project when the theoretical design is turned out into a working system. Thus it can be measured to be the most critical stage in achieving a successful new system and in giving the user, confidence that the new system will work and be effective.

The implementation stage involves vigilantscheduling, exploration of the existing system and it's constraints on implementation, designing of methods to realize and evaluation of changeover methods.

\section{MODULE DESCRIPTION: \\ 1. Server Module \\ 2. Client1 Module \\ 3. Client2 Module}

1. Server Module:In this unit, Admin have three sub unit. They are,

A.Upload Video: Here Admin can add a new video. It's used for user for viewing further collections.

B.User Details: Admin can view the user those have registered in this site.

C.Rate videos: This unit for avoiding unexpected videos from users. After accept or reject videos then only user tin view their own videos.

2. Client1Module: This unit, it contains the following sub unit and they are,

A.News Feed: Here user of this social site can view status from his friends like messages or videos.

B.Search Friends: Here they can search for a friends and send a request to them also can view their details.

C.Share Video:They can share videos with his friends by adding new videos also they share their status by sending messages to friends.

D.Update Details: In this Module, the user can update their own details.

Client2 Module:In this module, user can register their details like name, password, age,gender and then. Here the user can make friends by accept friend request or send friend request.

They can portion their rank by messages also share videos with friends and get comments from them. 


\section{CONCLUSION}

In this paper we will presented adaptive Computer Video streaming and sharing framework, called ACESCloud, which efficiently stores videos in the clouds (VC), and utilizes cloud computing to construct private agent (subVC) for each Computer user to try to offer "nonterminating" video streaming adapting to the changeability of link value based on the Scalable Video Coding technique. Also ACES-Cloud can further seek to provide "non-buffering" experience of video streaming by background pushing functions among the VB, subVBs and localVB of computer users. We evaluated the ACESCloud by prototype implementation and shows that the cloud computing technique brings significant improvement on the adaptivity of the computer streaming. The key factor of this paper is to verify how cloud computing can improve the transmission adaptability and prefetching for computer users. We ignored the cost of encoding workload in the cloud while implementing the prototype. As one vitalcomingwork, we will carry out large-scale implementation and with serious consideration on energy and price cost. In the future, we will also try to progress the SNS-based prefetching, and security issues in the ACES-Cloud.

\section{REFERENCES}

[1] IEEETRANSACTIONSONMULTIMEDIA,VOL.15,NO.4,JUNE2013.

[2] IEEE Communications Society subject matter experts for publication in the WCNC 2006 proceedings. 1-4244-0270-0/06/\$20.00 (c)2006 IEEE

[3] G.Wang and T. E.Ng, "The impact of virtualization on network performanceof amazon EC2 data center," in Proc. IEEE INFOCOM, 2010, pp. 1163-1171.

[4] T. Nguyen, N. Boukhatem, Y. Doudane, and G. Pujolle, "COPSSLS: A service-level negotiation protocol for the Internet," IEEE Commun. Mag., vol. 40, no. 5, pp. 158-165, May 2002.

[5] IEEE TRANSACTIONS ON MULTIMEDIA, VOL. 11, NO. 6 , OCTOBER 2009

[6] J. A. Colas et al., "Connecting ambient networks-Architecture and protocol design (release 1)," Ambient Networks Consortium, Deliverable D 3.2, Mar. 2005

[7] J. C. Chen, A. McAuley, V. Sarangan, S. Baba, and Y. Ohba, "Dynamic service negotiation protocol (DSNP) and wireless DiffServ," in Proc ICC 2002, New York, Apr. 2002.

[8] J. C. Fernandez, T. Taleb, N. Ansari, K. Hashimoto, N. Kato, and Y. Nemoto, "Dynamic QoS negotiation for next generation wireless communications systems," in Proc. WCNC 2007, Hong Kong, China, Mar. 2007

[9] Xiaofei Wang, Student Member, IEEE, MinChen, Senior Member, IEEE, Ted Taekyoung Kwon, Member, IEEE, LaurenceT. Yang, Senior Member, IEEE, and Victor C. M. Leung, Fellow, IEEE

\section{BIOGRAPHIES}

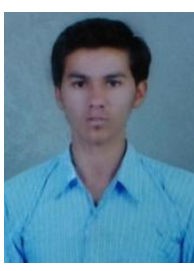

Dr.Panjabrao MSBTE

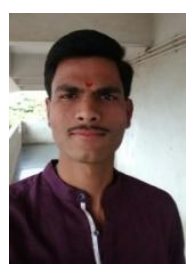

Kishor Ramesh Phapale is student of BE in Information Technology from Sinhgad Institute of Technology, Lonavala, Pune affiliated to AICTE under Savitribai Phule Pune University and Completed Diploma inComputer Technology in the year 2012 from Govt Polytechnic, Ahmadnagar, in

MSBTE.

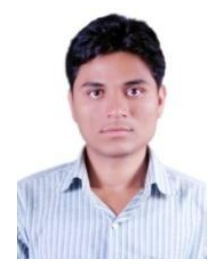

Roshan Sakharam Pawar is student of $\mathrm{BE}$ in Information Technology from Sinhgad Institute of Technology, Lonavala, Pune affiliated to AICTE under Savitribai Phule Pune University and Completed Diploma in Information Technology in the year 2012 from Dr.N.P. HiraniInstitute Polytechnic, Pusad, Yawatmal in MSBTE.

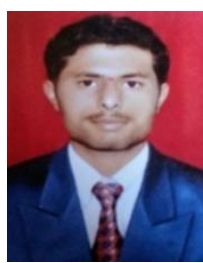

Sagar Deshmukh is is student of BE in Information Technology from Sinhgad Institute of Technology, Lonavala, Pune affiliated to AICTE under Savitribai Phule Pune University and Completed Diploma in Information Technology in the year 2012 from B.MPolytechnic,Solapur in MSBTE.

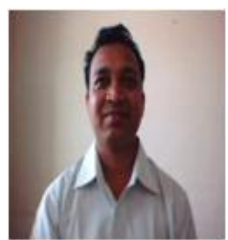

Prof. N. A. Dhawas

HOD \& Associate Professor

Department of Information Technology

Sinhgad Institute of

Technology,Lonavala student of BE in Information Technology from Sinhgad Institute of Technology, Lonavala, Pune affiliated to AICTE under Savitribai Phule Pune University and Completed Diploma in computer engineering in the year 2012 from Deshmukh Polytechnic, Amravati in 